\section{Orchestrating mitosis}

The anaphase-promoting complex APC/C is a key regulator, and regulation point, during mitosis, when events must happen in an ordered fashion to ensure that each daughter cell receives a correct set of chromosomes. By ubiquitinating a number of substrates once the chromosomes are properly aligned

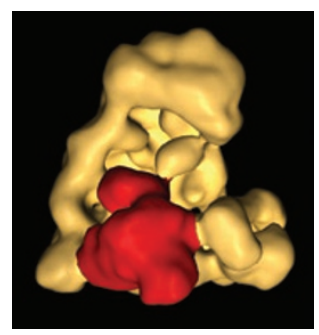
and attached to the spindle, the APC/C triggers the onset of chromosome separation. The APC/C is activated by Cdc 20 but inhibited by a complex called the MCC. By purifying different forms of the APC/C from checkpoint-arrested human cells, Stark, Peters and colleagues have now been able to gain insight into the structure and regulation of the APC/C. The authors isolated the apo-APC/C and the MCC-associated form of $\mathrm{APC} / \mathrm{C}$ and used single-particle EM to analyze their structures. By adding recombinant $\mathrm{Cdc} 20$ to the apo-APC/C, they could also examine the active form of the complex. The EM analyses show that the APC/C consists of a platform plus an 'arc lamp'-like region that adopts varying conformations in the apo complex. Using antibodies, the authors mapped the positions of particular components, and they argue that the repetitive-looking 'stem' of the arc lamp may be formed by the tetratricopeptide repeats present in certain APC/C components. The region to which Apc4 maps has a ring-like feature that may correspond to Apc4's predicted propeller-shaped WD40 repeats. MCC renders the APC/C more compact and conformationally stable, and seems to overlap with the region where density for Cdc20 resides, suggesting that its inhibition of the APC/C may result from directly interfering with $\mathrm{Cdc} 20$ binding. The authors also found that the MCC-bound form of the complex was slightly inhibited in its ability to bind the ubiquitin-conjugating enzyme UbcH10 but was more strikingly defective in substrate binding. These results provide important insights into the structural basis for the regulation of this complex. (Science 323, 1477-1481, 2009)

$S L$

\section{Digesting a switch}

When a DNA double-strand break (DSB) occurs, two related kinases, ATM and ATR, are activated, inducing a checkpoint that halts the cell cycle while the lesion is repaired. In mammals, ATM activation involves binding of the MRN complex to a DSB, whereas ATR activation involves binding of the single-stranded DNA-binding protein RPA to resected single-strand tails. As DSBs are often converted into tailed molecules, it was possible that a hand-off between ATM and ATR activities might occur. Shiotani and Zou have examined the basis for such a switch using nuclear extracts from $\mathrm{HeLa}$ cells. They find that ATM activation requires pairing of the ends of doublestranded DNA. The presence of single-strand overhangs (SSOs) reduces ATM activation by interfering with binding of the MRN complex. ATM activation is dependent on its binding to the double strand-single strand junction and not the SSO of a tailed DNA; furthermore, this junction needs to be at the end and not internal to the molecule. In vivo, SSOs are covered by RPA, which recruits ATR-interacting protein (ATRIP). As a linear duplex is progressively digested by an exonuclease to make an SSO in vitro, ATM activation is inhibited, while ATR activation is enhanced. But is it the single-strand tail itself, or activation of ATR by the RPA-ATRIP complex, that downregulates ATM? When the level of ATR was suppressed, ATM activation was still inhibited in damaged cells, suggesting that DSB resection was sufficient. Thus, although ATM is activated by resected DSBs,

Written by Angela K. Eggleston, Joshua M. Finkelstein, Maria Hodges \& Sabbi Lall as the resection proceeds, ATR activation becomes dominant. This would alter the downstream kinase targets and help move the repair process along. (Mol. Cell 33, 547-558, 2009)

$A K E$

\section{NMR in living cells}

Atomic-resolution structures of proteins in living human cells would link three-dimensional structural information to biological processes. In-cell NMR allows observations of the conformations and functions of proteins in living cells, but requires two problems to be overcome: how to label proteins so that they are detectable by NMR spectroscopy in human cells and how to collect an adequate data set without destroying the cell. Until now two approaches have been used to label proteins for NMR studies. The first is to grow bacteria on isotopically labeled medium. The second is to microinject labeled proteins into large cells such as Xenopus oocytes. Inomata et al. introduce a third technique: fusing cell-penetrating peptides to ${ }^{15} \mathrm{~N}$-labeled proteins. In the presence of pyrenbutyrate, the labeled target protein is taken into the cell, where the peptide is removed. This approach was so successful that the authors used it to study the intracellular dynamics of ubiquitin. They found that ubiquitin in cells was more dynamic and less structured than ubiquitin examined in vitro. The other major difficulty with in-cell NMR is the limited lifetime of cells within the NMR sample tube. NMR data-collection experiments usually take between one and two days, but Sakakibara et al. have shortened this to two to three hours by implementing a nonlinear sampling scheme, which differs from standard NMR sampling methods. They demonstrated the technique with three different proteins, one of which (FKBP12) forms specific complexes with externally added immunosuppressants-meaning that this technique might be useful for drug screening. In addition, they solved the structure of a putative heavy-metal-binding protein, TTHA1718. (Nature 458, 102-105 and 106-109, 2009)

$\mathrm{MH}$

\section{Don't you know you're toxic?}

Some transition metals, such as iron and copper, are essential for the catalytic activity of key metalloproteins, whereas others - for example, cadmium or silver — can be very toxic to both prokaryotic and eukaryotic cells. The acquisition and cellular uptake of transition-metal ions and their intracellular transport to specific protein targets are extremely complex processes, and the mechanisms by which cells import sufficient quantities of 'essential' transition-metal ions while keeping other, more toxic, transition metals out of the cell are not fully understood. Lewinson et al. recently reported the use of a cell-based 'metal tolerance' assay to characterize 18 prokaryotic P-type ATPases, proteins that actively transport metal ions across cellular membranes. The authors were able to definitively classify five of the P-type ATPases as 'exporters': two could restore zinc and cadmium tolerance to a metal-sensitive strain of Escherichia coli, and the other three increased tolerance to exogenously supplied copper and silver ions. None of the identified ATPases could nonselectively export all four transition-metal ions. The authors also identified a transition-metal 'importer,' HmtA, that was able to mediate the cellular uptake of copper and zinc- 'essential' transition metalsbut not cadmium or silver. Unraveling the exact mechanism by which HmtA achieves the observed selectivity for copper and zinc will require additional work, but it is noteworthy that HmtA homologs are present in several pathogens, suggesting that it may play a role in virulence. (Proc. Natl. Acad. Sci. USA, doi:10.1073/ pnas.0900666106; published online 5 March 2009) 